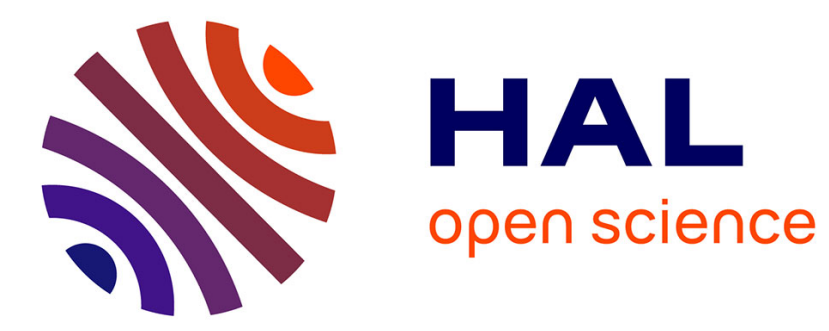

\title{
Numerical insight of a variational smeared approach to cohesive fracture
}

\author{
F. Freddi, F. Iurlano
}

\section{To cite this version:}

F. Freddi, F. Iurlano. Numerical insight of a variational smeared approach to cohesive fracture. Journal of the Mechanics and Physics of Solids, 2017, 98, pp.156-171. 10.1016/j.jmps.2016.09.003 . hal-02145287

\section{HAL Id: hal-02145287 \\ https://hal.science/hal-02145287}

Submitted on 2 Jun 2019

HAL is a multi-disciplinary open access archive for the deposit and dissemination of scientific research documents, whether they are published or not. The documents may come from teaching and research institutions in France or abroad, or from public or private research centers.
L'archive ouverte pluridisciplinaire HAL, est destinée au dépôt et à la diffusion de documents scientifiques de niveau recherche, publiés ou non, émanant des établissements d'enseignement et de recherche français ou étrangers, des laboratoires publics ou privés. 


\title{
Numerical insight of a variational smeared approach to cohesive fracture
}

July 15, 2016

\author{
F. Freddi ${ }^{1}$ and F. Iurlano ${ }^{2}$
}

${ }^{1}$ Department of Civil Environmental Engineering and Architecture, University of Parma, Parco Area delle Scienze 181/A, I 43124 Parma, Italy

${ }^{2}$ Institut für Angewandte Mathematik, Universität Bonn 53115 Bonn, Germany

\begin{abstract}
In the present paper we numerically investigate and validate a variational smeared model for cohesive crack, recently proposed and theoretically justified by $\Gamma$-convergence. To achieve this main goal, we first analyze the response of a bar subjected to traction. Possible solutions are discussed, reconstructing the classical cohesive fracture energy and its related stress-crack opening law through a backtracking procedure. Preliminary 2D investigations are also conducted by using a regularized version of the adopted formulation. This permits to explore the transition phase of the damage evolution and to determine the peculiarities of the model, such as mesh-objectivity and $\Gamma$-convergence as damage concentration is forced. Therefore, the numerical simulations confirm the analytical results and put the basis for further engineering applications and possible improvements of the model.
\end{abstract}

KEYwORDS: Cohesive fracture, Phase field model, Variational approach.

\section{Introduction}

Cohesive models in Fracture Mechanics are based on the idea that fracture energy is gradually released with the growth of the crack opening, reflecting the progressive weakening of the bound between the lips [10]. In contrast, in Griffith's brittle model the fracture energy is instantaneously dissipated at crack initiation [35], the surface energy is proportional to the crack area and there is no mechanical interaction between the fracture lips.

In the last years fracture problems have been revisited taking advantage of tools and techniques from the calculus of variations [16, 28, 24]. Although with some limits, a consistent and rigorous treatment of the subject has been built, so that numerical simulation and implementation have required appropriate methods. A standard approach consists in studying a regularized version of the fracture model.

Phase field models have recently found widespread popularity for simulating brittle crack propagation in a smeared manner $[15,14,39,41,26]$. Some phenomena, which are difficult to catch using discrete crack models, are easily captured by phase field models. This is the case of crack nucleation, propagation, and bifurcation, whose identification now requires no specific criterions. 
The analytical substrate is the $\Gamma$-convergence result by Ambrosio and Tortorelli [7], which yields the convergence of global minima as concentration of damage is forced. The original numerical approach [15] has been modified in order to avoid the problem of material overlapping and to describe specific failure modes [8], [37],[30], such as in the case of no-tension materials [31]. Extension to dynamics has been proposed in [17], [13]. Recently, the case of plastic material has been investigated in [32], [33]. After passing an energy barrier for slip activation, elastic strain energy is locally converted into plastic work on slip surfaces. Alternative formulations for elasto-plastic material based on the classical decomposition of strain in elastic and plastic parts can be found in [1], [4].

The counterpart of [7] in the cohesive context is represented by the recent work [20]. In the prior result [3], phase field type energies linear in the strain are used to approximate cohesive fracture; while in $[36,22,27]$ phase field models are shown to converge to energies with a linear surface density. Finally in [23] cohesive fracture in elasto-plastic materials is obtained as limit of gradient damage models coupled with plasticity.

In the present paper we simulate cohesive crack initiation and propagation using the regularized model introduced in [20]. The fracture process in stressed bodies is reproduced through the minimization of a two-field elliptic functional, in which the first field stands for the displacement within the solid, whereas the second one, here called phase field or damage, represents the transition between sound and completely broken material.

The model is numerically implemented using a standard finite-element discretization and adopting an alternate minimization algorithm coupled with a backtracking technique originally proposed in [14]. In such a way, we reconstruct the Barenblatt's cohesive energy and the stress-crack opening law in a 1D traction experiment. Particular attention is paid to the transition phase, for which the analytical study provides only partial results. It is interesting to notice that without a backtracking procedure the relevant curves get close to those corresponding to the homogeneous solution at moderate opening.

Irreversibility is quite a controversial issue in the cohesive fracture context and its discussion goes out of the aims of the present work. We refer to [16, 24] for a comprehensive analysis and a discussion of the relevant topics. Here we assume the standard damage irreversibility on the smeared functionals by requiring the monotonicity of the damage variable. It turns out that the stress-crack opening law is linear during unloading and reloading up to the maximal opening, where we recover the standard Barenblatt's law.

We also consider an alternative version of the phase field models [20] which is preferable from a computational point of view, and we compare its behavior with the original one. This formulation turns out to be effective in $2 \mathrm{D}$ experiments, for which no theoretical result is currently available.

The paper is structured as follows. In Section 2 we recall the phase field formulation for cohesive crack of [20] and we compare it with the case of brittle fracture [7]. In Section 3 we provide the details for the numerical treatment and the implementation. A 1D traction experiment is deeply investigated in Subsection 4.1, with and without the backtracking procedure. As the size of the damaged region shrinks, $\Gamma$-convergence is numerically demonstrated for two versions of the formulation. Finally a 2D investigation is conducted in Subsection 4.2. Finally, conclusions and perspectives are illustrated in 
Section 5 .

\section{Formulation in case of antiplane shear}

Let $\Omega \subset \mathbb{R}^{d}$ be a bounded open set with Lipschitz boundary representing the crack-free reference configuration of an elastic body. We assume that, due to suitable external forces and boundary conditions, the body developes a $(d-1)$-dimensional crack and undergoes a displacement $u$ out from the crack. In the antiplane cohesive model, the evolution of this process is described by an energy which is sum of a bulk term, the energy of the crack-free part, and of a surface term, the energy spent to separate the fracture lips. The former depends on the strain $\nabla u$, the latter on the crack set $J_{u}$ and on the amplitude $[u]$ through an appropriate density function $g: \mathbb{R} \rightarrow[0,+\infty]$.

The variational formulation of the model involves the space of special functions with bounded variation $S B V$, whose properties can be found in [6]. The stationary cohesive energy reads as

$$
\int_{\Omega} \frac{c}{2}|\nabla u|^{2} d x+\int_{J_{u}} g(|[u]|) d \mathcal{H}^{d-1}
$$

where $u \in S B V(\Omega), g$ is nondecreasing, concave, $g(0)=0, g(s) \rightarrow \gamma$ as $s \rightarrow+\infty$, and the slope of $g$ in 0 is $\sigma_{c} \in(0,+\infty)$, being $\sigma_{c}$ the maximum stress. The model is then completed by taking into account the action of external forces and the boundary conditions.

The direct method of the calculus of variations, used to guarantee the existence of global minimizers for the functional under consideration, requires lower semicontinuity and coercivity properties in the ambient space. Regrettably, the energy (2.1) is not lower semicontinuous nor coercive in $S B V$. In order to overcome this difficulty we replace $S B V$ with the space of generalized functions with bounded variation $G B V$ (see [6]) and apply the direct method to the relaxed functional

$$
\mathcal{F}(u):=\int_{\Omega} \psi(|\nabla u|) d x+\int_{J_{u}} g(|[u]|) d \mathcal{H}^{d-1}+\sigma_{c}\left|D^{c} u\right|(\Omega),
$$

where $\nabla u, J_{u}$, and $[u]$ are still well-defined though in a generalized sense, $D^{c} u$ is the Cantor derivative of $u$, and $\psi(s):=c s^{2} / 2$ for $s \leq(\gamma /(2 c))^{1 / 2}, \psi(s):=\sigma_{c} s-\gamma / 4$ otherwise. The last term in (2.2) can be interpreted as a micro-crack energy with zero bulk and surface energies, see [16].

This approach is supported by the fact that in general a functional and its relaxation share the same infimum. In particular in 1D and under suitable hypotheses on $\psi, g$, boundary conditions, and forces, a global minimizer $\bar{u}$ of (2.2) belongs to $S B V$ and $|\nabla \bar{u}| \leq(\gamma /(2 c))^{1 / 2}$, so that $\psi(|\nabla \bar{u}|)=|\nabla \bar{u}|^{2}$ (see $\left.[18]\right)$.

Next we present the variational approximation of (2.2) proposed in [20, Theorem 3.1]. We introduce an auxiliary field $v$, representative of the damage state within the material. It takes value 1 where the material is sound and value 0 where the material is fully disaggregated. For definition and properties of $\Gamma$-convergence we refer to [21].

Given two infinitesimal sequences $0<\eta_{\delta}<<\delta$ and $c, \gamma \in(0,+\infty)$, we consider the functionals $F_{\delta}$ defined by

$$
F_{\delta}(u, v):=\int_{\Omega}\left(\frac{c}{2}\left(\eta_{\delta}+f_{\delta}^{2}(v)\right)|\nabla u|^{2}+\gamma\left(\frac{(1-v)^{2}}{4 \delta}+\delta|\nabla v|^{2}\right)\right) d x
$$


for $(u, v) \in H^{1}(\Omega) \times H^{1}(\Omega)$ and $0 \leq v \leq 1$ a.e., and by $+\infty$ otherwise in $L^{1}(\Omega) \times L^{1}(\Omega)$, where

$$
f_{\delta}(z):=\min \left\{1, \delta^{1 / 2} \frac{z}{1-z}\right\}, \quad f_{\delta}(1):=1 .
$$

By [20, Theorem 3.1] as $\delta \rightarrow 0$ the functionals $F_{\delta} \Gamma$-converge in $L^{1}(\Omega) \times L^{1}(\Omega)$ to the functional $F(u, v):=\mathcal{F}(u)$ defined in $(2.2)$, if $u \in G B V(\Omega)$ and $v=1$ a.e., $F(u, v):=$ $+\infty$ elsewhere. The surface density $g$ can be determined by the following one-dimensional minimum problem

$$
g(s):=\inf _{(\alpha, \beta)} \int_{0}^{1} \sqrt{\frac{c \gamma}{2} \beta^{2}\left|\alpha^{\prime}\right|^{2}+\gamma^{2}\left(1-\beta^{2}\right)\left|\beta^{\prime}\right|^{2}} d r,
$$

where $\alpha, \beta \in H^{1}((0,1))$ satisfy $0 \leq \beta \leq 1, \alpha(0)=0, \alpha(1)=s, \beta(0)=\beta(1)=1$. In particular $g$ is nondecreasing, subadditive, $g(0)=0$, the slope of $g$ in 0 is $\sigma_{c}:=(c \gamma / 2)^{1 / 2}$, and the asymptotic limit as $s \rightarrow+\infty$ is $\gamma$ (see [20, Proposition 4.1]).

Roughly speaking, in $1 \mathrm{D}$ when $|[u]|$ is small the damage variable $v$ is close to 1 in a $\delta$-neighborhood of the fracture point, and $\nabla v$ gives a negligible contribution to the fracture energy. Therefore $f_{\delta}(v) \sim \delta^{1 / 2} v /(1-v)$, the first two terms in (2.2) balance out and

$$
g(|[u]|) \sim \sqrt{\frac{c \gamma}{2}} f_{\delta}(v)(1-v) \frac{|[u]|}{\delta} \sim \sigma_{c}|[u]| .
$$

When $|[u]|>>0, v$ is forced to pass from 1 to 0 in a $\delta$-neighborhood of the fracture point, and the contribution of the first term in (2.2) becomes negligible. Therefore the last two terms in (2.2) balance out and

$$
g(|[u]|) \sim \gamma,
$$

recovering the Ambrosio-Tortorelli regime [7], see (2.7) below. For intermediate values of $|[u]|$ all terms in (2.2) give a non-negligible contribution. The relation between the value $|[u]|$ and an optimal profile $v$ is more delicate and requires further investigation.

We remark that actually more general $f_{\delta}$ can be considered. As an example one can replace $z /(1-z)$ in $(2.4)$ by any $\varphi \in C^{0}([0,1),[0,+\infty))$ nondecreasing, such that $\varphi^{-1}(0)=\{0\}$ and $\varphi(z)(1-z) \rightarrow 1$ as $z \rightarrow 1$. In this case in $(2.5)$ one obtains $\varphi^{2}(\beta)(1-\beta)^{2}$ in place of $\beta^{2}$.

Another possible generalization is to smoothen the truncation in (2.4), this will be preferable in 2D numerical experiments (see Subsection 4.2). Indeed, let us define $\hat{f}_{\delta} \in$ $C^{1}([0,1])$ linearly near $z=1$ :

$$
\hat{f}_{\delta}(z):= \begin{cases}\delta^{1 / 2} \frac{z}{1-z} & \text { if } z \leq \bar{z}_{\delta}:=\frac{1-\delta^{1 / 2}}{1+\delta^{1 / 2}} \\ a_{\delta}\left(z-\bar{z}_{\delta}\right)+b_{\delta} & \text { otherwise in }[0,1],\end{cases}
$$

with

$$
a_{\delta}:=\frac{\left(1+\delta^{1 / 2}\right)^{2}}{4 \delta^{1 / 2}}, \quad b_{\delta}:=\frac{1-\delta^{1 / 2}}{2} .
$$

In Fig. 1 a the functions $f_{\delta}(z)$ and $\hat{f}_{\delta}(z)$ are plotted for two values of $\delta$. It clearly emerges that the difference between the two functions vanishes as $\delta \rightarrow 0$. One can check 
that the functionals $\hat{F}_{\delta}$, defined as in $(2.3)$ with $f_{\delta}$ replaced by $\hat{f}_{\delta}, \Gamma$-converge to $F$, with $g$ given exactly by (2.5) (see [20, Remark 3.2]).

In the next sections we validate the analytical results of [20] by using either $f_{\delta}$ or $\hat{f}_{\delta}$. Numerical implementations involving $f_{\delta}$ require some tricks to compensate for the lack of differentiability and to permit fracture initiation. Nevertheless, they give slightly more precise results in $1 \mathrm{D}$ with respect to those relying on $\hat{f}_{\delta}$. In $2 \mathrm{D}$ we adopt $\hat{f}_{\delta}$ in place of $f_{\delta}$ since it is computationally less expensive, see Section 4.2.

Ambrosio-Tortorelli's formulation. We conclude this section recalling the $\Gamma$ convergence result obtained by Ambrosio and Tortorelli in [7] for brittle fracture. In this case the approximating energies are functionals $G_{\delta}$ defined as in (2.3), with $f_{\delta}(v)$ replaced by $v$. They prove in [7] that $G_{\delta} \Gamma$-converges to the Griffith energy $G$

$$
G(u, v):=\frac{c}{2} \int_{\Omega}|\nabla u|^{2} d x+\gamma \mathcal{H}^{d-1}\left(J_{u}\right)
$$

if $u \in G S B V(\Omega)$ and $v=1$ a.e., $G(u, v):=+\infty$ elsewhere in $L^{1}(\Omega) \times L^{1}(\Omega)$.

Fixed $\delta$ small, it can be seen that the damage $v_{\delta}$ obtained minimizing $G_{\delta}$ under suitable boundary conditions has the following profile

$$
v_{\delta}(x) \sim 1-e^{-\frac{\left|x-x_{0}\right|}{2 \delta}},
$$

for $x$ close to some point $x_{0}$, and $v_{\delta} \sim 1$ otherwise.

The quasi-static version of the Ambrosio-Tortorelli result has been proposed by Giacomini in [34]. Regrettably, no evolutionary result is currently available in the cohesive context, not even in the antiplane case.

a)

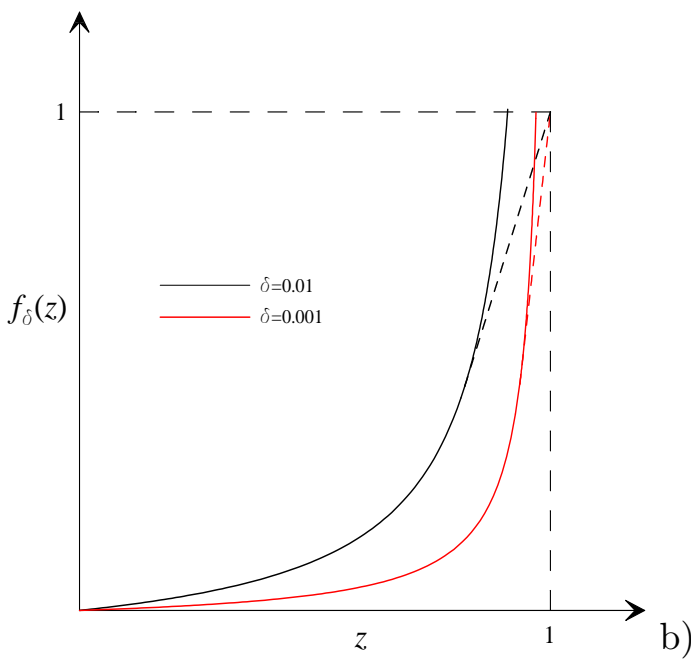

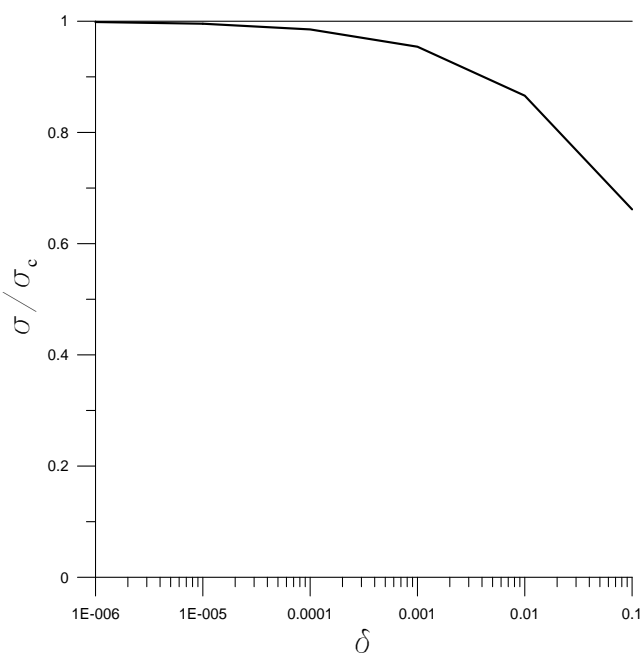

Figure 1: (a) Plot of $f_{\delta}(z)$ (continuous line) and $\hat{f}_{\delta}(z)$ (dashed line) for $\delta=0.01,0.001$; (b) plot of the maximum stress as a function of $\delta$.

\subsection{Damage homogeneous solutions in 1D}

In a simple 1D example we compare the behavior of spatially homogeneous solutions of the phase field problem in the cohesive case (2.4) and in the brittle case. 
Let us consider a strained bar of unitary length, fixed at $x=0$, undergoing the displacement $u(1)=u$ at the end $x=1$, and homogeneous Neumann boundary conditions $v^{\prime}(0)=v^{\prime}(1)=0$ for the damage field. For simplicity we introduce $\zeta(z)$ which stands for $z^{2} /(1-z)^{2}$ (cohesive regime, neglecting the truncation in (2.4)) or $z^{2}$ (AmbrosioTortorelli brittle regime). Denoting the strain and stress within the bar respectively by $\varepsilon:=u^{\prime}$ and $\sigma:=\zeta(v) \varepsilon$, we impose the following set of equations for the general coupled phase field problem

$$
\begin{aligned}
& \sigma^{\prime}=0 \\
& \frac{c}{2} \zeta^{\prime}(v) \varepsilon^{2}-\gamma \frac{1-v}{2 \delta}-2 \gamma \delta v^{\prime \prime}=0 .
\end{aligned}
$$

The former represents the balance equation, the latter is the evolution equation for $v$ in the quasi-static case $(\dot{v}=0)$.

Let us consider a spatially homogeneous solution $v(x) \equiv v_{\text {hom }}$ to the equations. It follows that the strain must be constant $\varepsilon(x) \equiv \varepsilon_{0}=u$. One can check that both in the cohesive regime $\zeta(z)=z^{2} /(1-z)^{2}$ and in the brittle regime $\zeta(z)=z^{2}$, to each given $\varepsilon_{0}$ corresponds a unique solution $v_{\text {hom }}$, and $v_{\text {hom }} \searrow 0$ as soon as $\varepsilon_{0} \nearrow+\infty$. In particular, in the brittle regime the unstrained bar $\left(\varepsilon_{0}=0\right)$ is the only configuration in which $v_{\text {hom }}=1$, so that the elastic domain is trivial. Instead, in the cohesive regime we compute the actual amount of damage through the truncation $f_{\delta}^{2}\left(v_{\text {hom }}\right)=\min \left\{1, \delta v_{\text {hom }}^{2} /\left(1-v_{\text {hom }}\right)^{2}\right\}$, therefore we find a linear stress-displacement relation for $\varepsilon_{0}$ small.

a)
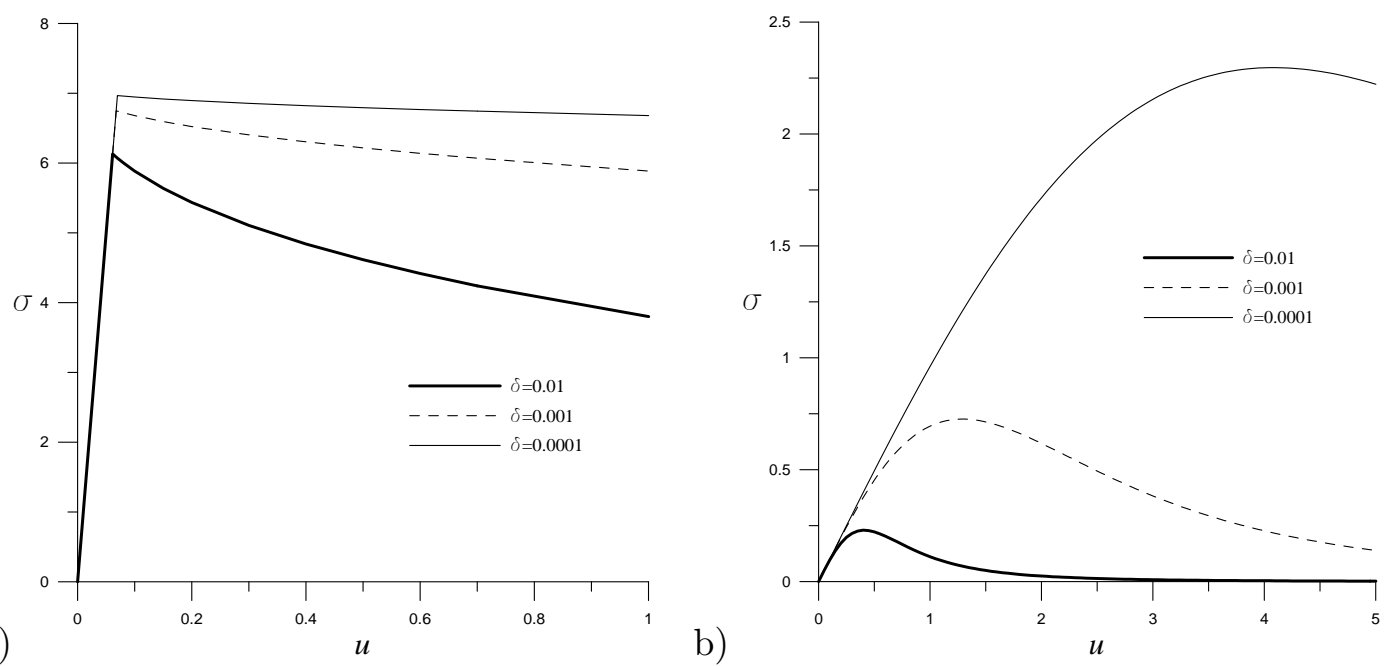

Figure 2: Homogenous solution: global response of a bar in terms of stress-displacement for different values of the parameter $\delta$ obtained adopting $\gamma=1$ and $c=100$. (a) Cohesive case; (b) brittle case.

The numerical study of (2.8) is presented in Fig. 2. Since the bar is originally undamaged, the solution of the evolution problem (2.8) is characterized by an elastic phase and a damaging phase. The stress as a function of the applied displacement is drawn in Fig. 2a for three values of the parameter $\delta$. The elastic regime is guaranteed up to a critical value of the strain, corresponding to the maximum admissible stress. In this phase the truncation in (2.4) is active and a linear stress-strain relation $\sigma=c \varepsilon_{0}$ holds. 
When the strain exceeds the critical value, the damaging process affects the response and a softening branch appears. Such branch asymptotically approaches the horizontal line $\sigma=\sigma_{c}$ as $\delta \rightarrow 0$. We also note that the stress peak converges to the value $\sigma_{c}$ when $\delta \rightarrow 0$, as shown in Fig. 1b.

In the brittle case the unique homogeneous solution to (2.8) can be explicitly computed. The response, drawn in Fig. $2 \mathrm{~b}$ for different values of the parameter $\delta$, does not present an elastic phase for any prescribed displacement different from zero. The bar undergoes a stress-hardening phase up to the stress peak, that in this case is given by $9 / 16 \sqrt{c \gamma /(6 \delta)}$. Subsequently, the bar is in stress-softening regime up to complete rupture. In particular the stress peak goes to infinity as $\delta \rightarrow 0$.

Finally, we recall that the stress does not concentrate in the 1D case. As a consequence, in the Griffith regime both the maximum stress and the critical strain become infinite as $\delta \rightarrow 0$; this is in accordance with the theoretical fact that it is not possible to initiate a fracture without a preexisting flaw. In the cohesive regime the stress peak is always bounded, but an infinite strain is needed to create a complete fracture (equivalently to the case of infinitely cohesive jumps, see [16]). In both cases, brittle and cohesive, an infinite energy is necessary to create a fracture within the solid. We conclude that the homogeneous solutions cannot be global minima of the corresponding functionals.

\subsection{Irreversibility}

While in the Griffith's approach the concept of fracture point is rather simple, in the Barenblatt's model it has to be specified. Indeed, there is a particular state during the softening phase, here called pre-fracture according to [25], in which failure is initiated, strain starts to localize and non-null bridging forces can be transmitted across the lips of the crack. At this point the physical continuity of the material is still guaranteed and it will be lost only at total fracture, when the solid is no more able to transmit bridging forces. Only at this stage a fracture point in the sense of Griffith is created, material rupture is irreversible, and the dissipation is complete. In all subsequent deformation processes the cohesive energy remains constant and the cohesive force is zero.

Working with the smeared functionals in the brittle context three different choices are commonly adopted to impose irreversibility. A threshold for the variable $v$ is introduced in order to define the completely fractured zones [14]. Once this threshold has been overcome, irreversibility is enforced and the phase field parameter is set $v=0$. Such condition, numerically convenient with respect to the damage monotonicity $\dot{v} \leq 0$ of [34], is reasonable in this context in view of the definition of Griffith fracture point. Alternatively, the maximum value of the driven force in the damage evolution equation is considered [5]. This allows to obtain damage monotonicity from thermodynamic considerations.

Differently, in the cohesive zone model the transient phase of stress transmission plays a crucial role, so that memory of the partial crack must be kept before coalescence occurs. Thus, the monotonicity of $v$ seems to be the proper irreversibility condition capable of taking into account the degradation of the process zone. In Subsection 4.1 we show which irreversibility condition is consequently inherited by the sharp 1D model. 


\section{$3 \quad$ Numerical details}

\subsection{Extended formulation}

The definition in (2.3) can be extended to the case of vector-valued displacement as in [27]. Given $(\mathbf{u}, v) \in H^{1}\left(\Omega, \mathbb{R}^{d}\right) \times H^{1}(\Omega), 0 \leq v \leq 1$ a.e., we set with abuse of notation

$$
F_{\delta}(\mathbf{u}, v):=\frac{1}{2} \int_{\Omega}\left(\eta_{\delta}+f_{\delta}^{2}(v)\right) \mathbb{C}\left[\nabla^{s} \mathbf{u}\right] \cdot \nabla^{s} \mathbf{u} d \mathbf{x}+\frac{\gamma}{2} \int_{\Omega}\left(\frac{(1-v)^{2}}{4 \delta}+\delta|\nabla v|^{2}\right) d \mathbf{x},
$$

where $\eta_{\delta}<<\delta, \mathbb{C}:$ Sym $\rightarrow$ Sym is the elastic tensor of the sound material, $\nabla^{s} \mathbf{u}$ is the symmetric part of the gradient $\nabla \mathbf{u}$, and $f_{\delta}$ is defined in (2.4). In this case the Cauchy stress $\mathbf{T}$ reads as

$$
\mathbf{T}=\left(\eta_{\delta}+f_{\delta}^{2}(v)\right) \mathbb{C}\left[\nabla^{s} \mathbf{u}\right] .
$$

As in Section 2, we define $\hat{F}_{\delta}$ replacing $f_{\delta}$ by $\hat{f}_{\delta}$. Notice that, neglecting $\eta_{\delta}$, one recovers the stress of a sound elastic material when $f_{\delta}(v)=1$. One expects that regions where $f_{\delta}(v)$ is small tend to concentrate in bands of thickness of order $\delta$, where strain localizes $\left(\left|\nabla^{s} \mathbf{u}\right|\right.$ becomes very large).

Regrettably, no rigorous $\Gamma$-convergence result is currently available in this general case. The reason is essentially technical, related to the lack of appropriate lower semicontinuity and relaxation results in the limit functional space $(G) B D(\Omega)$ of functions with bounded deformation [42]. As a consequence, even proposing a candidate limit model is not trivial. Anyway, one expects that the limit bulk density will be again linear at infinity, and that the surface density will depend on the opening $[\mathbf{u}]$ and on the normal $\nu_{\mathbf{u}}$ to the jump set through some periodic cell formula. Moreover all terms of the functional (3.1) will contribute to the crack energy, differently from the brittle context in which essentially the last two terms play a role. Numerical experiments involving functionals (3.1) will be presented in Subsection 4.2.

In what follows we refer to the first term of (2.3) and of (3.1) as bulk (or elastic) contribution and to the last two terms as surface (or fracture) contribution.

\subsection{Numerical treatment}

Our numerical approximation is based on a discretization in both space and time. The minimization problem for $F_{\delta}$ is numerically solved using an incremental procedure. Let the non-dimensional loading (and time-like) parameter $t$ be increased from 0 to the final value $T$ with $n$ finite increments of amplitude $t_{0}=T / n$. Accordingly, $\mathbf{u}_{t}$ and $v_{t}$ represent the displacement and the damage field evaluated at time $t$ respectively.

The numerical treatment of the functional $F_{\delta}$ is not a trivial task and some of the difficulties are analogous to those encountered in the case of brittle fracture. The main obstacles are listed below.

- The functional is non-convex, so that classical algorithms guaranteeing convergence to global minimizers fail. Moreover, appropriate methods available in literature in general only ensure convergence to stationary points. In order to overcome these difficulties we adopt an alternate minimization algorithm with backtracking, details are given below. 
- The functional, due to truncation in (2.4), is not Gateaux differentiable and not convex with respect to the phase field variable $v$. It also presents null driving force when the truncation is active. These issues involve major disadvantages, concerning the minimization in $v$ and the trigger of the damage mechanism. Such problems can be overcome by neglecting the truncation when minimizing with respect to $v$. In such a way differentiability and convexity are recovered and rupture process can initiate. The drawback is no guarantee that the alternate minimization procedure constructs at each time step a sequence with decreasing total energy. Alternatively, the linearized function (2.6) can be adopted without any restriction.

- According to [16], the discretization error has to be carefully taken into account for a proper analysis of the results. An accurate estimation of the discretized surface energies requires the element size $h$ to be much smaller than the regularization parameter $\delta$. The discretization error numerically found in the case of brittle fracture [16] is confirmed in the present formulation. For linear finite elements discretization the fracture energy is overestimated by a factor that depends on the size $h$ of the discrete element and on the parameter $\delta$

$$
\gamma \rightarrow \gamma\left(1+\frac{h}{4 \delta}\right)
$$

This overestimation is taken into account in the numerical simulation presented in Section 4 .

- The evolution is numerically evaluated at discrete steps. In view of this, we prevent material self-healing by adopting the irreversibility condition

$$
v_{t}(\mathbf{x}) \leq v_{t-1}(\mathbf{x}), \forall \mathbf{x} \in \Omega .
$$

The numerical scheme is based upon an alternate minimization algorithm which, in short, consists in solving a series of minimization sub-problems on $\mathbf{u}$ at fixed $v$ and viceversa on $v$ at fixed $\mathbf{u}$ up to convergence. The procedure is detailed in Algorithm 1. In particular, the energy functional is quadratic in $u$ and the elastic sub-problem reduces to the solution of a linear system of equations. The only inconvenience is due to the strongly spatially varying stiffness parameter which leads to linear algebra difficulties in computing the system solution.

The minimization on $v$ at fixed $\mathbf{u}$ is reduced to solve a nonlinear unconstrained problem and to project a posteriori the solution on the set of admissible space to enforce the irreversibility condition. Alternatively, the irreversibility condition has been applied with an augmented Lagrangian approach following the numerical strategy illustrated in [43] without noticeable difference.

Since alternate minimization relies only on stationarity conditions for $\mathbf{u}$ and $v$, it cannot be guaranteed to converge to a global minimum of (2.3). The aim of the backtracking algorithm, proposed and discussed in depth in [14] in the brittle framework, is to derive a further necessary condition for optimality and an algorithmic way to check it. Although there is no proof that the back-tracking algorithm converges to a global minimum, at each time step it forces to compare, and possibly redefine, the evolution given by the alternate minimization with those obtained in the previous times. 


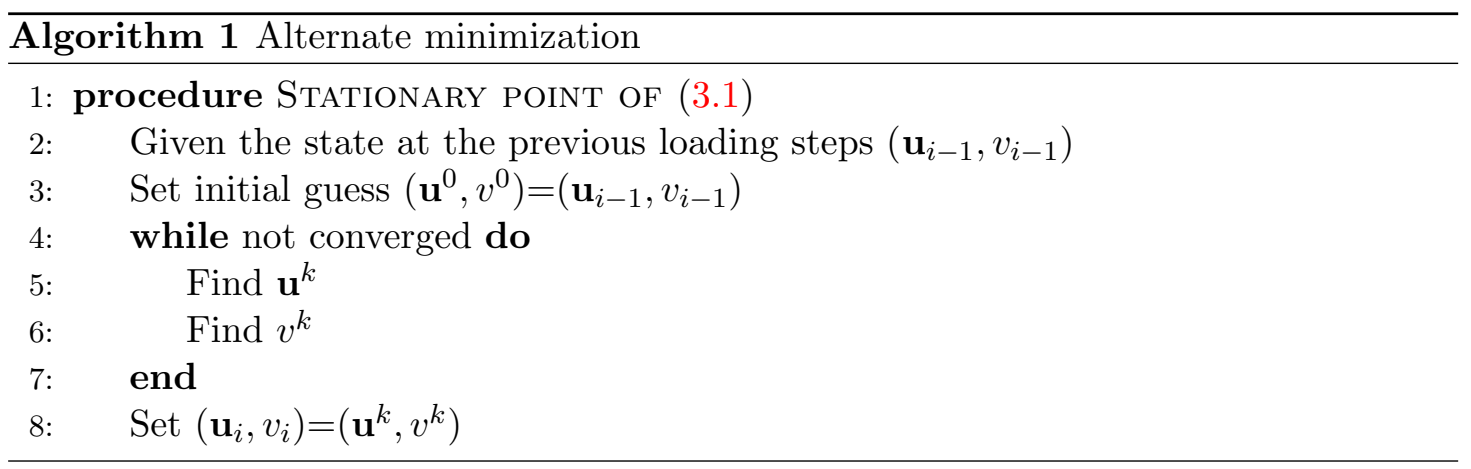

Consider a monotonically increasing loading $u_{0} t$ and two loading steps $t_{l}$ and $t_{j}$ such that $t_{l} \leq t_{j}$. If the pair $\left(\mathbf{u}_{j}, v_{j}\right)$ is admissible for (3.1) at time $t=t_{j}$, then $\left(t_{l} / t_{j} \mathbf{u}_{j}, v_{j}\right)$ is admissible at time $t=t_{l}$. Therefore, if $\left(\mathbf{u}_{l}, v_{l}\right)$ is a global minimizer at time $t=t_{l}$ then $F_{\delta}\left(\mathbf{u}_{l}, v_{l}\right) \leq F_{\delta}\left(t_{l} / t_{j} \mathbf{u}_{j}, v_{j}\right)$ and for any $0 \leq j \leq N$ we have

$$
F_{\delta}\left(\mathbf{u}_{l}, v_{l}\right) \leq \frac{t_{l}^{2}}{t_{j}^{2}} F_{\delta}^{b}\left(\mathbf{u}_{j}, v_{j}\right)+F_{\delta}^{s}\left(v_{j}\right), \forall 0 \leq l \leq j,
$$

where $F_{\delta}^{b}$ and $F_{\delta}^{s}$ denote respectively the bulk and the surface contribution in (3.1).

In Algorithm 2, condition (3.3) is checked against all previously computed time steps upon convergence of the alternating minimization algorithm. If a violation is detected, the time evolution backtracks to the relevant step keeping the current configuration as initial guess. A refined version of the algorithm has been recently proposed in [38].

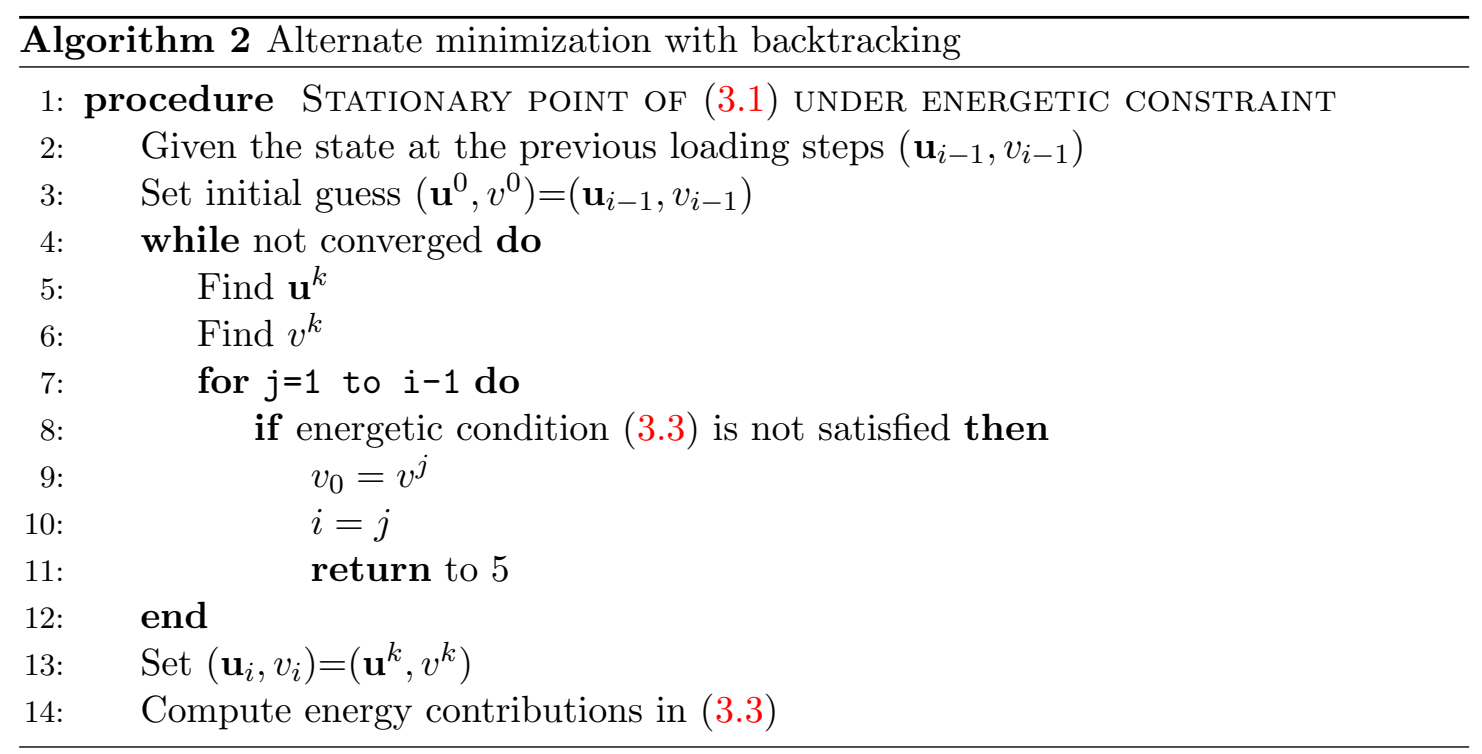

As in [14] and [30], the iteration loop is required to stop when the change in norm of the damage field descends a certain tolerance.

The parameter $\eta_{\delta}$ in (3.1) has been introduced in [7] to theoretically ensure the existence of a global minimizer for the smeared energy. It is often kept in numerical simulations to prevent numerical instability, though it is not strictly necessary (see for example [12]). Accordingly, in all calculations presented in this paper $\eta_{\delta}=0$ is assumed. 
The minimization problem has been numerically solved using a finite element method, implemented in a specifically conceived program based upon the Open Source package deal.II [9].

\section{Numerical Examples}

The scope of this section is to illustrate the response of the models (2.3) and (3.1) during a quasi-static evolution through representative examples. Firstly, a basic one-dimensional bar in traction is investigated. Subsequently, fracture propagation in a bi-dimensional notched specimen is simulated. Reference is made to Sections 2 and 3.1 for the notation used here.

\subsection{1d case}

In the following, we describe the evolutionary solution to the traction problem for a one-dimensional homogeneous bar.

Let $\Omega:=[0, L]$, so that $\partial \Omega=\{0, L\}$. We follow the evolution in the time interval $[0, T]=[0,1]$, assuming that the left extremity at $x=0$ is clamped, while a displacement $u=u_{0} t$ is imposed at $x=L$. Here $u_{0}$ is a reference length and $t \in[0,1]$ is a nondimensional loading parameter. Moreover, condition $v=1$ is assigned at $x \in\{0, L\}$ instead of adopting an enlarged logical domain. This condition is imposed to recover a correct value for the surface energy. Indeed, it forbids the development of fractures exactly at the boundary, although they are free to appear at a small distance. This fact may well interpret the confining effects offered in a real experimental set-up by glued supports.

The adopted material parameters are $c_{1}=100 \mathrm{MPa}$ for the Young's modulus, $c_{2}=1$ $\mathrm{Nmm}^{-1}$ for the fracture toughness, $L=1 \mathrm{~mm}$ for the length of the bar and the parameter $\delta=0.01 \mathrm{~mm}$. The time interval is divided into 1000 equally spaced time steps while $u_{0}=1 \mathrm{~mm}$. The domain is uniformly discretized into mono-dimensional linear finite elements such that $\delta / h=5$. This permits to have the same discretization error due to (3.2) for all simulations. Finally, if not explicitly declared, all the following simulations are performed adopting the backtracking algorithm described above.

Fig. 3a shows the stress-displacement curve obtained from FEM calculation. We observe two main phases of the cohesive fracture formation. Up to the displacement $u_{c}$, corresponding to the stress peak, we have a non-fractured state with a nearly uniform degradation along the bar. A damaged (pre-fractured) state characterizes the bar when $u>u_{c}$ up to complete decohesion, in which strain and damage clearly localize in a portion of the bar. In this phase cohesive forces appear and vanish as the deformation increases. The unloading-reloading path is also outlined; it follows a linear path. Complete fracture in the sense of Griffith's model can be achieved as $u \rightarrow \infty$.

We also investigate the energetic evolution in Fig. 3b, where the total energy, as well as its bulk and surface contributions (in a Griffith sense), are plotted as functions of the displacement. Note that in the displacement interval $\left[0, u_{c}\right]$ the evolution follows the elastic deformation: the bulk contribution moves from a convex to a concave behavior. Moreover, note that the truncation in (2.4) allows to obtain a bulk contribution which is quadratic near the origin. The total energy together with the surface contribution grow 


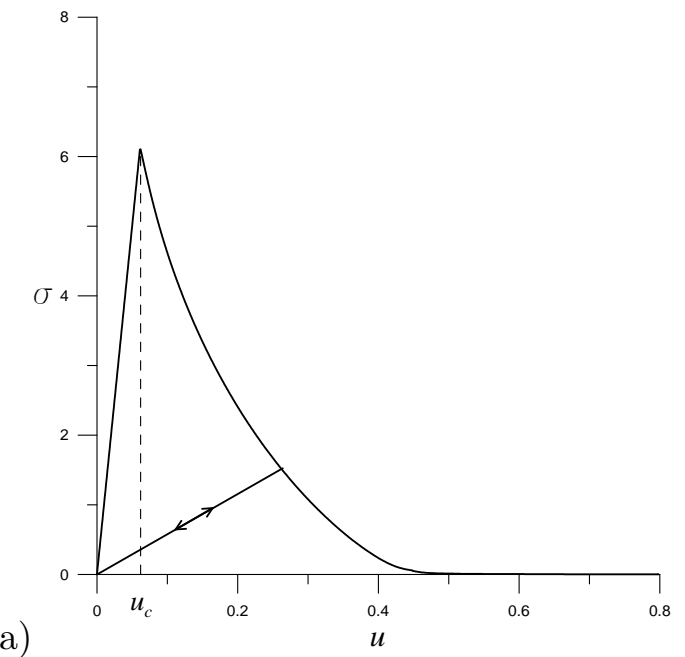

a)

b)

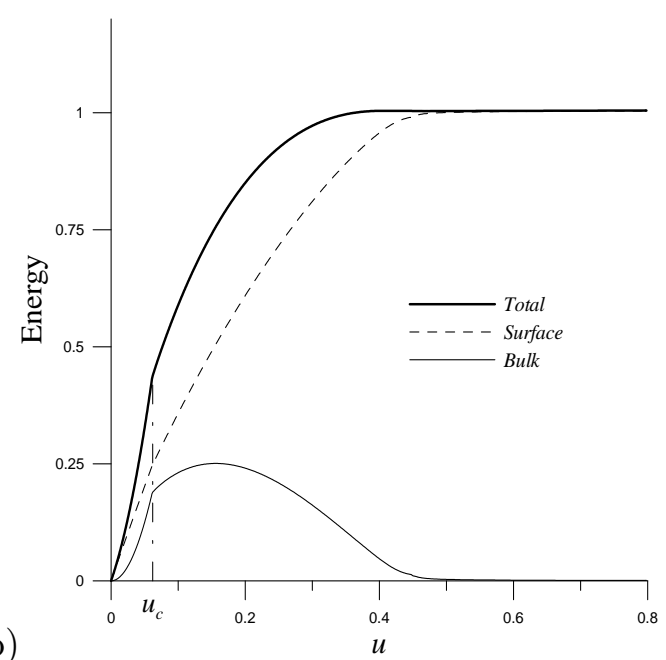

Figure 3: Bar in traction: (a) Stress as a function of the imposed displacement; (b) total energy as a function of the displacement: bulk contribution and surface contribution.

asymptotically up to the value $\gamma$, while the bulk counterpart vanishes. Even, the total energy moves from convex to concave evolution at $u=u_{c}$.

a)

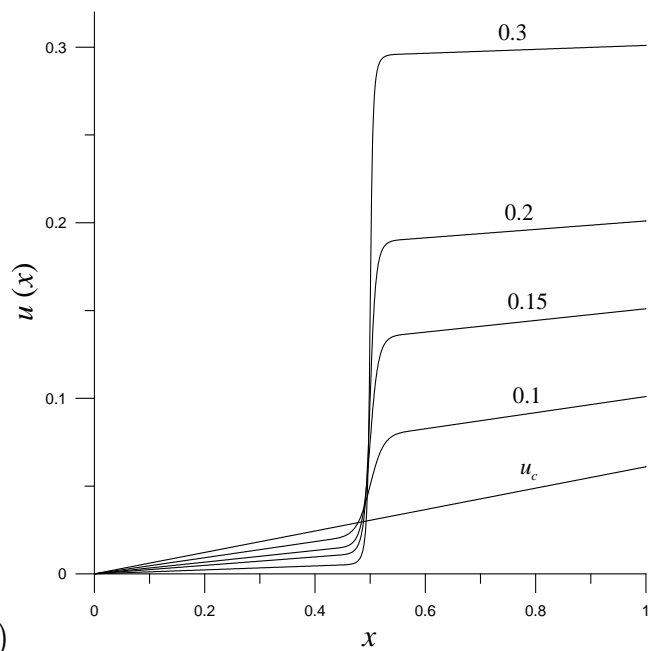

b)

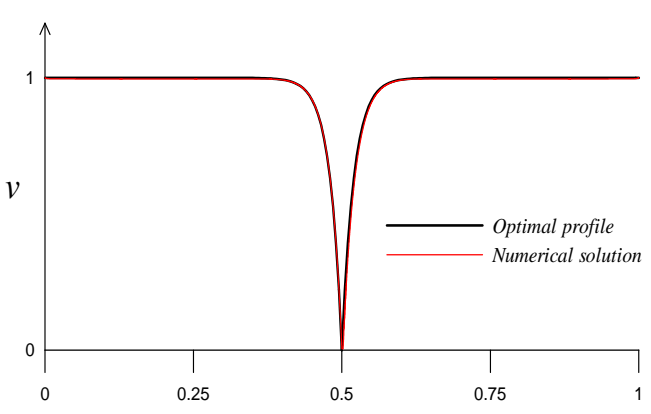

Figure 4: Bar in traction: (a) distribution of the displacement along the bar for different values of the imposed displacement; (b) profile of $v$ for completely damaged bar $(u=0.8$ $\mathrm{mm}$ ) and comparison with optimal profile of (2.7).

The two phases previously described are very clear in Fig. 4a where the displacement $u(x)$ is plotted along the bar for different values of the imposed displacement. Up to $u_{c}$ the bar undergoes linear elastic deformations. At this stage the elastic deformation is no more energetically convenient. Then, the pre-fracture phase starts in the central portion of the bar, in which a crack is opened, and a non-null bridging force is transmitted across the damaged region. As the imposed displacement increases, the deformation strongly localizes in this damaged zone thus exhibiting high displacement gradient. 
The physical continuity of the bar is supposed to be lost only at total fracture, when the jump amplitude reaches the critical value and the bridging force drops to zero. When this occurs a fracture point in the sense of Griffith is created. For the value of the applied displacement $u=0.8 \mathrm{~mm}$, representative of almost fractured phase, the profile of $v$ along the bar is plotted in Fig. $4 \mathrm{~b}$. In the middle of the bar $x \approx 0.5 \mathrm{~mm}$ the phase field parameter $v$ reaches the value $\cong 5 . e-3$.

The profile of $v$ obtained from the numerical solution is in a very good agreement with the optimal profile of (2.7), added in Fig. 4b. In this sense the phase field approach accurately approximates the sharp formulation.

a)

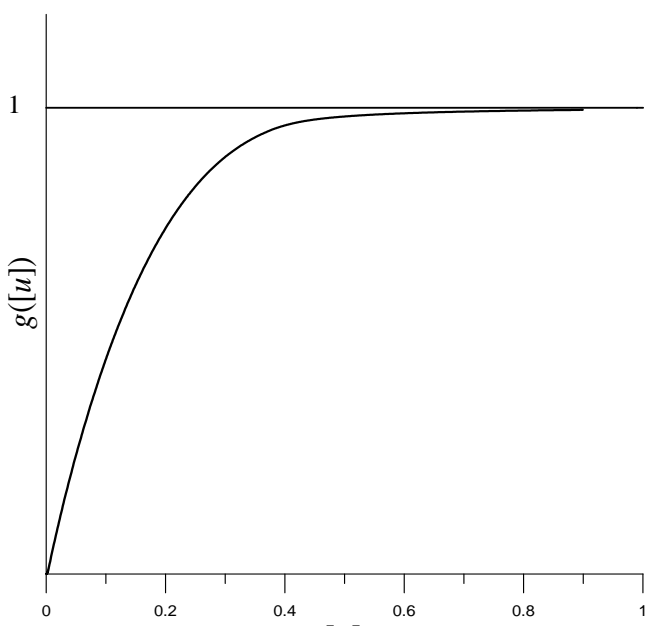

$[u]$

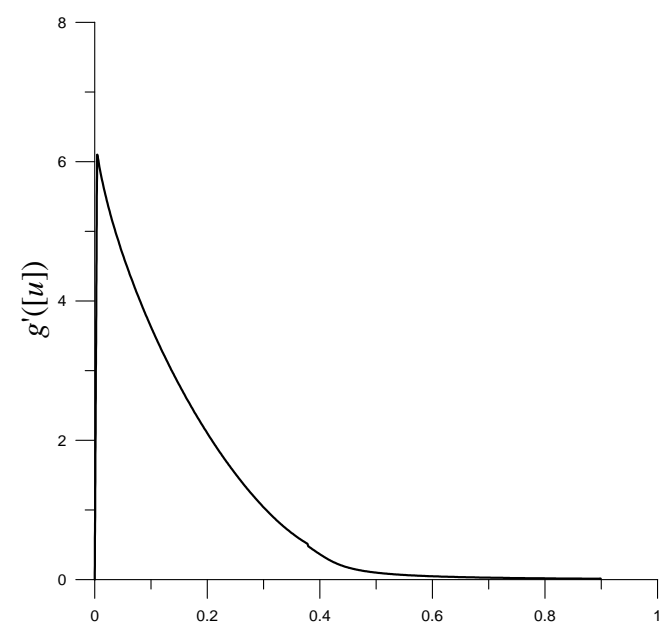

b)

Figure 5: Bar in traction: (a) graph of the cohesive energy density $g([u])$; (b) graph of the bridging force $g^{\prime}([u])$.

Finally, the cohesive energy density $g([u])$ is evaluated by numerical manipulation of the solution at points $x=0.5 \pm 0.03 \mathrm{~mm}$. The two points are just around the zone where high displacement gradient concentrates and the deformation recovers linear distribution. Firstly, the bridging force $g^{\prime}([u])$ transmitted across the crack of amplitude $[u]$ is obtained by the discrete solution, and then $g([u])$ is calculated by numerical integration of $g^{\prime}([u])$. Accordingly with the cohesive fracture theory, $g([u])$ is a monotonic non-decreasing function of the jump amplitude such that

$$
\gamma=\lim _{[u] \rightarrow \infty} g([u]) .
$$

Except for the initial linear portion which is due to the truncation (see $(2.4)), g^{\prime}([u])$ turns out to be continuous and decreasing, and it is a convex function of $[u]$; in such a case the energy is said sub-quadratic [25]. The initial ascending branch of $g^{\prime}([u])$ is commonly adopted in sharp cohesive models for interface problems [2], [29].

Now, a $\Gamma$-convergence study with respect to the parameter $\delta$ is carried out for this one-dimensional structure. In particular, in Fig. 6 a the stress-displacement curves obtained with $\delta$ ranging from 0.1 to $0.0001 \mathrm{~mm}$ are represented. In order to keep the convergence analysis consistent, a fixed ratio $h / \delta$ is adopted thus to have the same theoretical overestimation of the surface contribution (3.2) for each simulation. The initial 

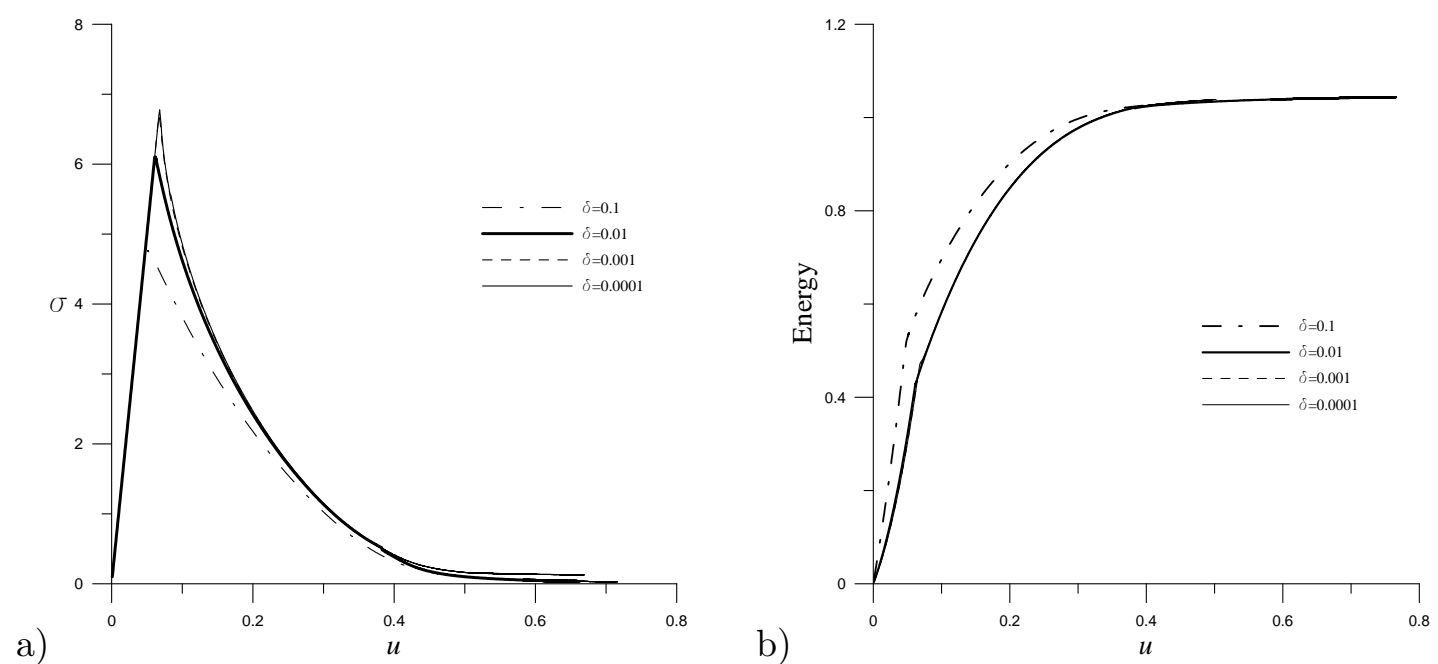

Figure 6: Bar in traction: $\Gamma$-convergence results for different values of the parameter $\delta$. (a) Stress as a function of the imposed displacement; (b) total energy as a function of the displacement.

purely linear elastic branch, which is due to the truncation in (2.4), results $\delta$ independent. The stress peak, as previously outlined in Fig. 1b, increases as $\delta$ decreases up to an asymptotic value. The softening branches, except for the initial part in the case $\delta=0.1$, present nearly coincident paths.

The total energy evolutions are drawn in Fig. 6 b. The cases $\delta=0.01,0.001,0.0001$ $\mathrm{mm}$ present perfect agreement. Differently, the case $\delta=0.1 \mathrm{~mm}$ highlights an energetic level higher than the other simulations in the initial part of the evolution. This is due to the fact that the length of the bar $L$ is too short for such $\delta$, so that the diffusion of damage affects the entire bar. The present numerical results indicate that, when the internal length scale $\delta \rightarrow 0$, the smeared energies corresponding to the phase field models converge towards the sharp cohesive fracture energy. The differences between the three curves cannot be graphically appreciated.

Let us replace now the function (2.4) by the regularized expression (2.6). The same $\Gamma$-convergence analysis is conducted neglecting the case $\delta=0.1 \mathrm{~mm}$, too large for the adopted bar length. The initial response is no more linearly elastic as clearly illustrated by Fig. 7a, where the stress as a function of the imposed displacement is plotted for different values of $\delta$. In fact, no elastic threshold is present in such a formulation.

The stress peak converges to the limit value $\sigma_{c}$ as $\delta$ decreases. After the peak, a small drop in the equilibrium path occurs vanishing as $\delta \rightarrow 0$. Such drop is due to the backtracking algorithm, which within a single time step goes back to recover a reduced value of $v$. Afterwards, the softening branches are equivalent to those obtained by adopting the original expression (2.4).

In Fig. 7b the total energy evolutions for the case at hand are depicted. The behavior is similar to that observed in Fig. 6b. The difference between the curves as $\delta$ varies is so limited that cannot be graphically appreciated. We conclude that even in this case numerical simulations confirm the $\Gamma$-convergence of the smeared functionals $\hat{F}_{\delta}$ to the sharp energy (2.2). 

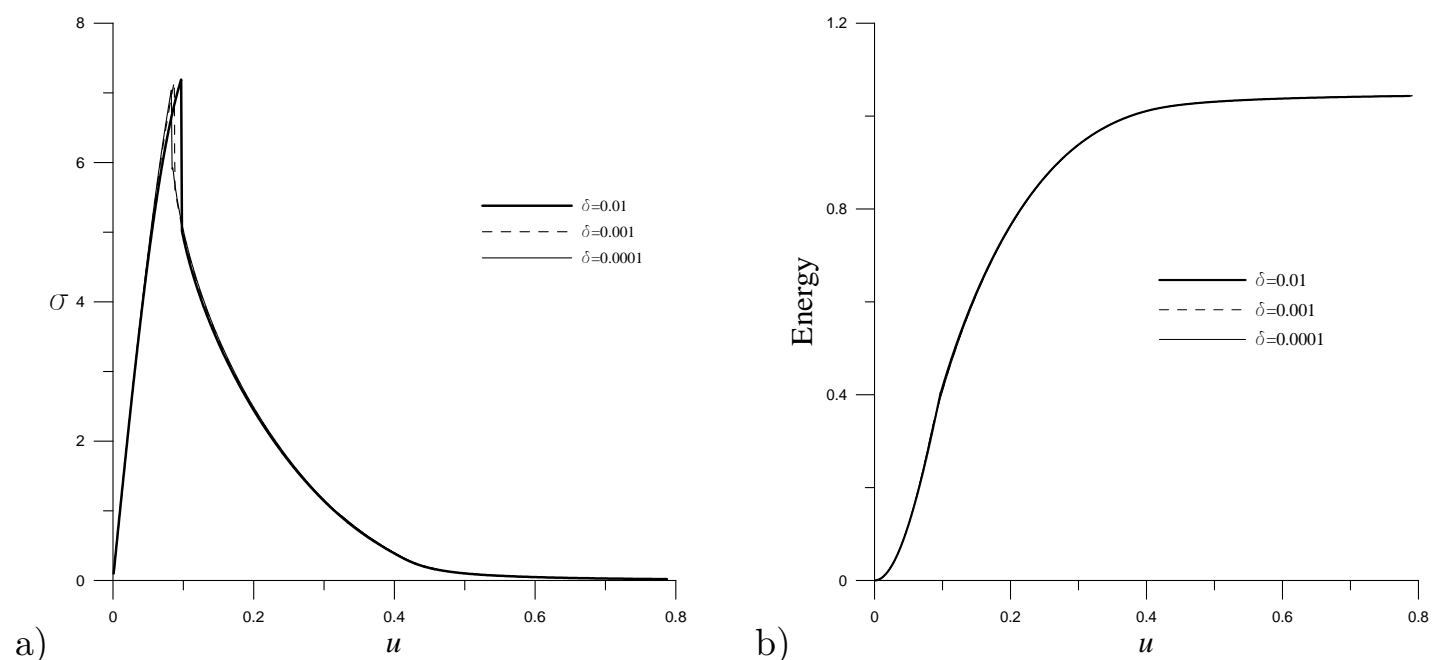

Figure 7: Bar in traction: $\Gamma$-convergence results for different values of $\delta$ obtained adopting $\hat{f}_{\delta}$. (a) Stress as a function of the imposed displacement; (b) total energy as a function of the displacement.

\subsubsection{Global minima vs stationary points}

The $\Gamma$-convergence simulations illustrated in Fig. 6 are repeated without adopting the backtracking algorithm. In Fig. 8 the stress and the energy are plotted as functions of the imposed displacement. For $\delta=0.1,0.01 \mathrm{~mm}$ no difference can be appreciated between the results of Fig. 6 and 8. Whereas, the evolution of the system changes after the peak for $\delta=0.001,0.0001 \mathrm{~mm}$. This phenomenon is rather clear in the stress-displacement graphs of Fig. 8a. Once that the linear elastic phase is exhausted, an almost horizontal plateau is followed for a certain amount of displacements; this is evident for $\delta=0.0001 \mathrm{~mm}$ and just outlined for $\delta=0.001 \mathrm{~mm}$. This portion of solution is exactly the homogenous solution that is described in 2.1 and is slightly reprised in Fig. 8a. After that, due to bifurcation of the numerical solution induced by numerical instability, a sudden partial failure occurs without a bridging phase. Finally, the equilibrium path merges in a softening branch.

It is important to underline that in these two cases $\delta=0.001,0.0001 \mathrm{~mm}$ we actually observe an evolution along critical points that certainly are not global minimizers: the homogeneous solution is not energetically favourable. The algorithm chooses the critical point which is the closest to the initial configuration, even if other options, more convenient from an energetic point of view, are available. This equilibrium path is particularly sustained by the physical idea of laziness of a body: it does not completely upset its configuration passing through energetic barriers, if a stable configuration can be obtained with less energetic expense. This condition is exacerbated in the $1 \mathrm{D}$ case, that cannot account for stress concentration. A similar behavior can be found in brittle fracture, where the solution deviates from the homogeneous deformation at an energetic level that strongly overestimates the stress peak [16].

From the energetic viewpoint, when the elastic deformation ceases to be a critical point the domain exhibits diffuse damage and the total energy continues to grow linearly up to the brittle jump. After that, the energy asymptotically converges to $\gamma$, see Fig. 8b. 

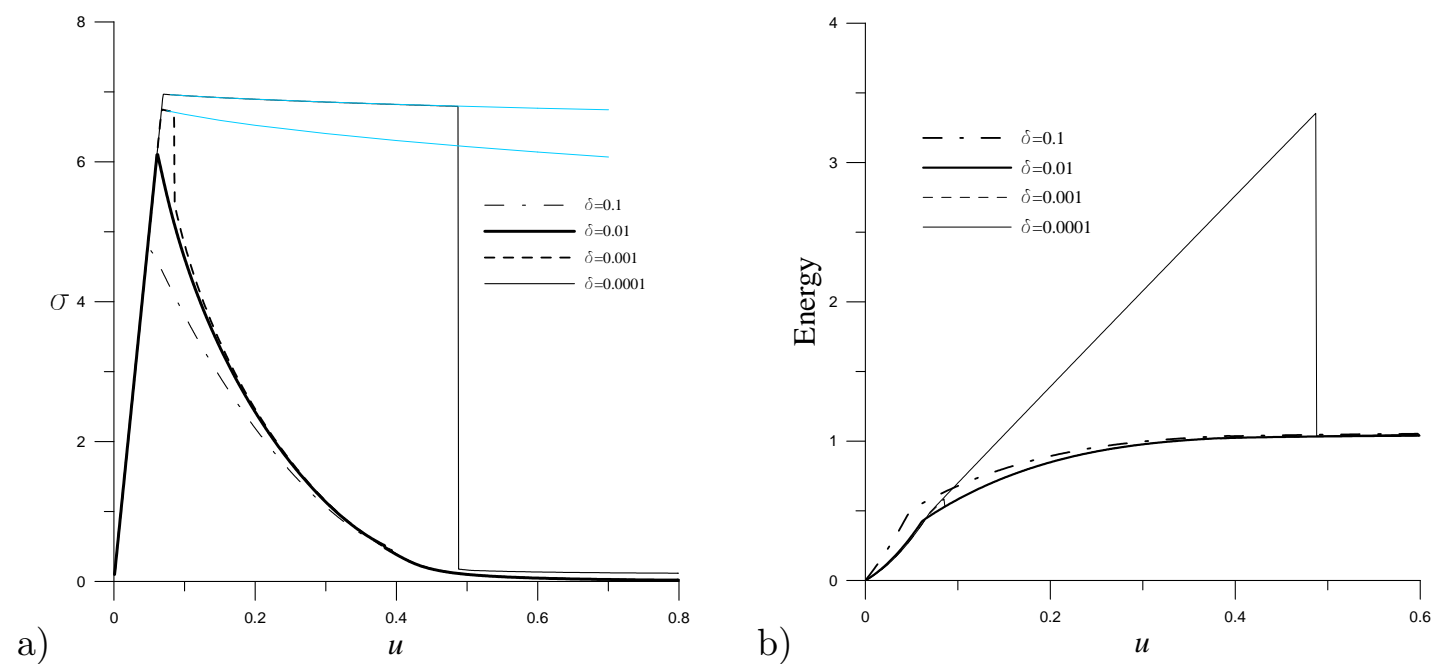

Figure 8: Bar in traction: $\Gamma$-convergence for different values of $\delta$ obtained without backtracking algorithm. (a) Stress as a function of the imposed displacement; (b) total energy as a function of the displacement.

\section{$4.22 \mathrm{~d}$ example}

We now challenge the formulation in a simulation of a two-dimensional cohesive fracture evolution. The simulations are performed only for a qualitative purpose since, as we have already mentioned, theoretical results are currently not available.

Let us consider the square plate of side $b$ represented in Fig. 9, with a V-notch of length $a$ along the left vertical side. The element is analyzed in plane strain condition and is loaded by applying opposite vertical displacements on the bases according to Fig. 9, while the vertical borders and the notch are unconstrained and stress-free. The horizontal bases are left free to expand. This setup may be representative of a tensile strain-driven test with lubricated loading platens. The material is assumed to be homogeneous and isotropic, so that $\mathbb{C}=2 \mu \mathbb{I}+\lambda \mathbf{I} \otimes \mathbf{I}$, where $\mu$ and $\lambda$ are the Lame's elastic constants.

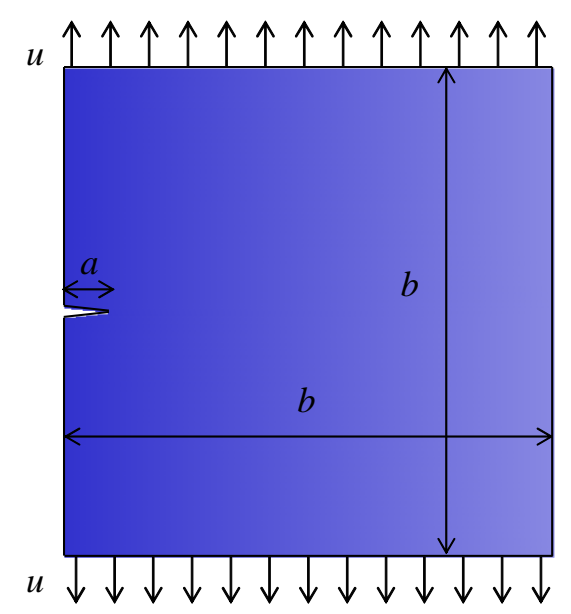

Figure 9: V-notched specimen subject to traction: setup and boundary conditions. 
The geometrical and material parameters used in the numerical simulation are: $b=1$ $\mathrm{mm}, a=0.1 \mathrm{~mm} \lambda=121153 \mathrm{MPa}, \mu=80769 \mathrm{MPa}, \gamma=0.1 \mathrm{Nmm}^{-1}, \delta=0.01 \mathrm{~mm}$. The computation is performed in a monotonic displacement-driven context with constant displacement increments $\triangle u=0.00001 \mathrm{~mm}$, being $u$ the vertical component of the imposed displacement. In the numerical discretization, we adopt a non-structured, antisymmetric, and inhomogeneous finite element mesh composed of 14048 quadrilaterals. Bilinear interpolations are used for the displacement and the damage field. In view of the horizontal symmetry, smaller finite elements are distributed close to the candidate crack path, in the center of the domain. In this portion the size of the element is $h \approx 0.4 \delta$.

The simulation is performed adopting the linearized function (2.6), already tested in the $1 \mathrm{D}$ case and computationally less expensive than (2.4). Indeed, due to the high variation of the function $v^{2} /(1-v)^{2}$ when $v \approx 1$, a very fine mesh is mandatory in the whole domain at the beginning of the damaging process, thus increasing the computational expense. The irreversibility condition for $v$ is taken into account.
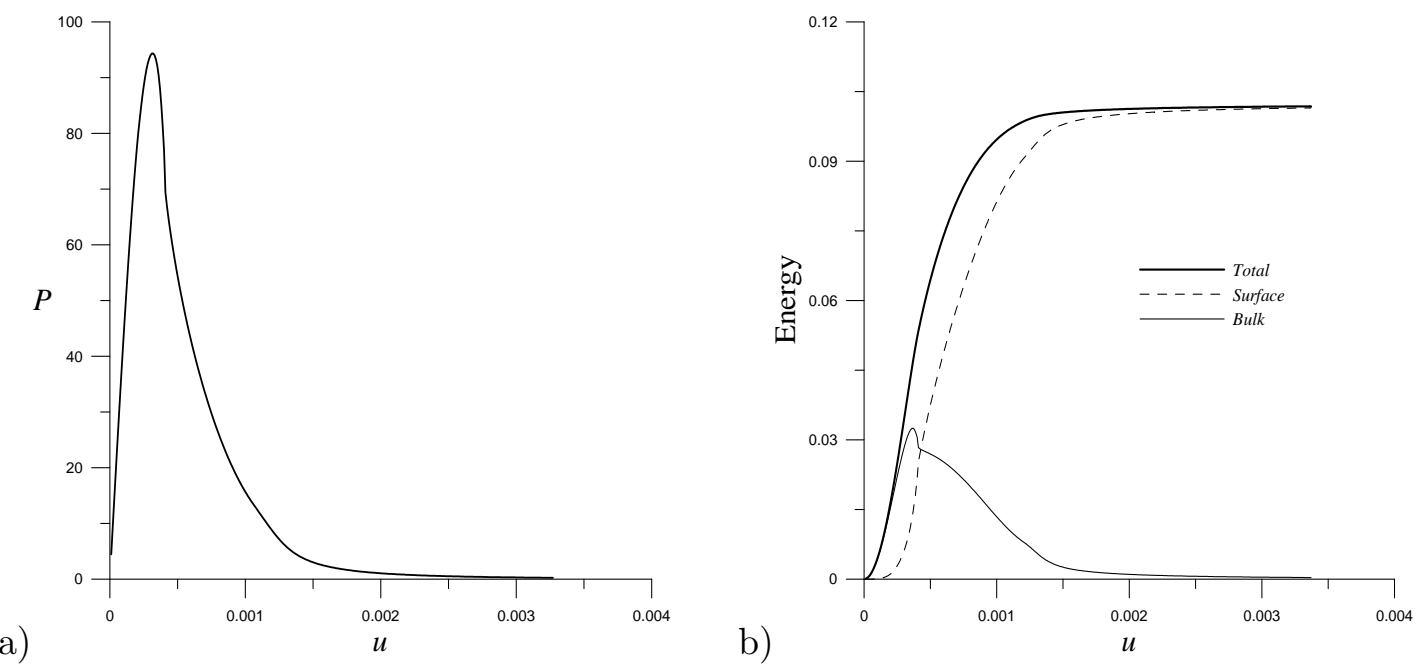

Figure 10: V-notched specimen: (a) Load (per unit width) as a function of the vertical displacement $u$; (b) total energy as a function of the displacement: bulk contribution and surface contribution.

The load $P$ calculated at upper basis is plotted in Fig. 10a as a function of the applied displacement $u$. The numerical solution clearly indicates a smooth transition from the almost linear elastic state of the ascending path to the softening regime of the descending branch once that the peak has been passed.

The total energy is drawn in Fig. 10b as a function of the applied displacement $u$. Such energy is composed of two contributions, here called bulk and surface as specified in Subsection 3.1, that are plotted in the same figure. It is again clear that the damaging process is characterized by an energy transfer from the bulk contribution to the surface counterpart. When the displacement is sufficiently large, the surface contribution of the energy grows asymptotically to the measure of the fractured part of the body (up to the factor $\gamma$ ). In this case the value of the energy is slightly overestimated for two main reasons: the discretization error (3.2) and the lack of elastic threshold. The latter, coupled with the irreversibility condition, leads the term $(1-v)^{2}$ in $\hat{F}_{\delta}$ (see Subsection 
3.1) to give non-negligible energetic contributions also on the non-fractured areas, being $v<1$ everywhere within the domain.

The resulting crack patterns at different stages of the deformation are illustrated in Fig. 11. The damaging process begins at the notch, where stress concentration occurs, and propagates horizontally up to affect the entire width of the specimen. As the displacement increases damage becomes more and more evident.

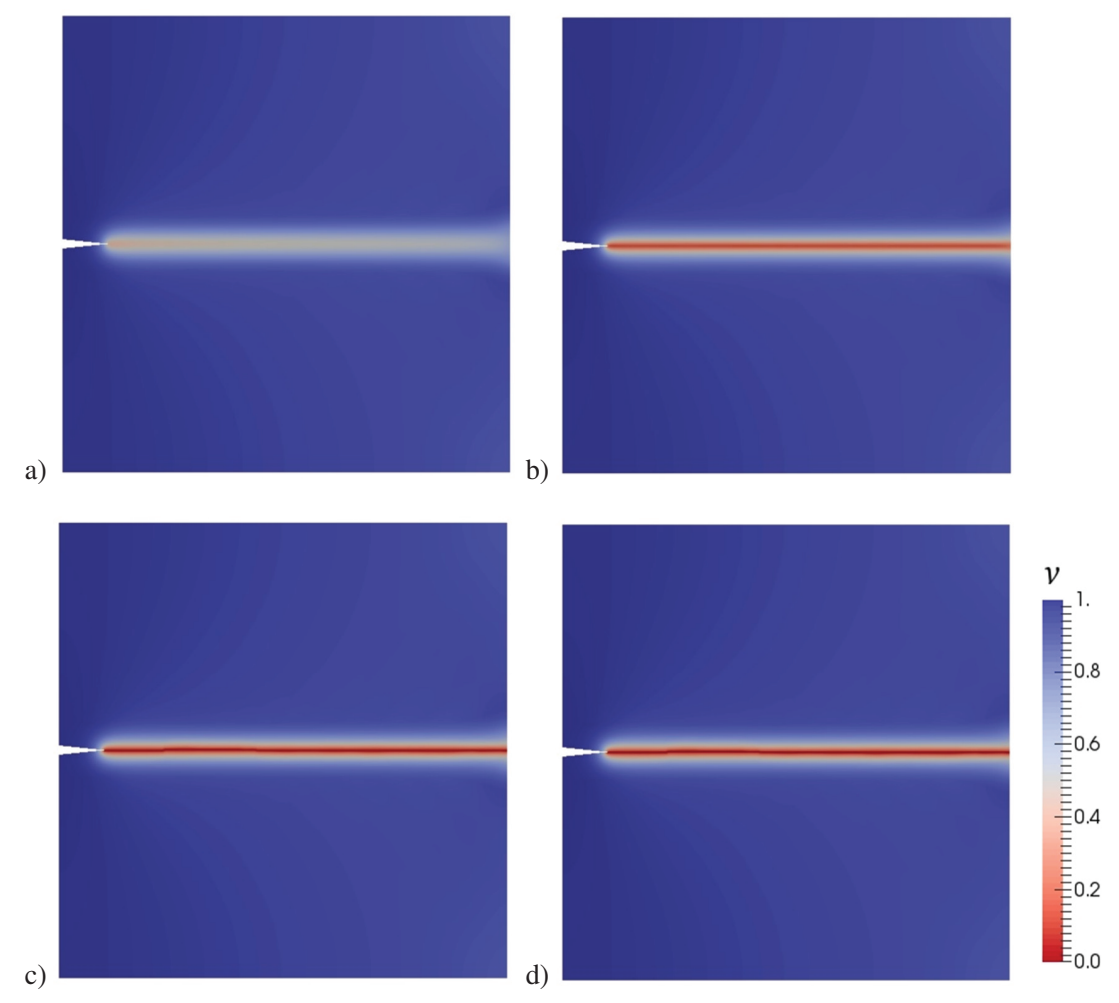

Figure 11: Map of the field $v$ for different values of the imposed displacement: (a) $u=0.0005$, (b) $u=0.001$, (c) $u=0.0015$, (d) $u=0.002$.

Localisation of deformations and mesh dependence of damage models have been studied extensively [11], [19]. In static problems the local equilibrium equations lose ellipticity near the onset of softening. Therefore deformation and damage grow, then localise in a line or, in finite element analyses, in the narrowest band that can be captured by the spatial discretization. Upon refinement of the discretization the predicted fracture energy tends to zero. In order to check mesh objectivity, simulations above are repeated with two globally refined meshes obtained by splitting into four element each cell. This procedure is applied twice so that three meshes are considered: mesh 1 (14048 elements), mesh 2 (56192 elements) and mesh 3 (224768 elements). For comparison purposes, the load-displacement responses for all cases are compiled in a single graph 12a. The dissipated energy is defined as the area underneath the force-displacement curves.

As evidenced in Fig. 12a the dissipated energy in the crack formation is clearly mesh insensitive. The structural responses are almost identical, ensuring the control of the energy dissipation regardless of the mesh refinement. Minor differences in the last portion of the softening branch can be attributed to the energetic error due to (3.2). Accordingly, 
the bigger is the element size the higher is the error estimation of the fracture energy. The comparison of structural responses in Fig. 12a leads to the conclusion that results are mesh-independent.

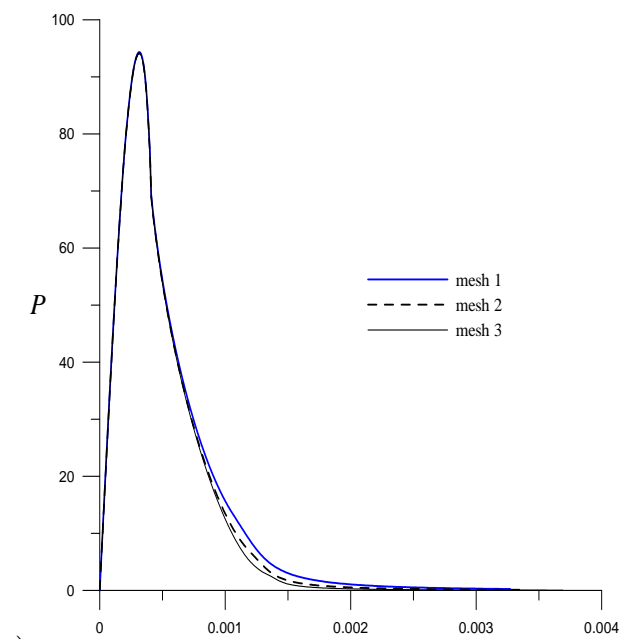

a)

Figure 12: V-notched specimen: (a) Load (per unit width) vs. vertical displacement $u$ for different meshes, (b) Load (per unit width) vs. vertical displacement $u$ for different values of the parameter $\delta$.

Finally, the influence of parameter $\delta$ on the solution has been investigated. The load is plotted in Fig. 12b as a function of the vertical displacement for different values of $\delta$. The global response, as for the 1D example, is not affected by the value of the parameter $\delta$. As might be expected, for a fixed mesh as in the case of our simulation, the error due to (3.2) leads to an energy overestimation which increases accordingly to a decrement of $\delta$.

\section{Conclusion and perspectives}

A recent variational formulation for cohesive crack [20] has been numerically assessed through representative examples.

Despite its simplicity, the one-dimensional setting has allowed us to give a quantitative description of the material behavior both from the constitutive and energetically point of view. In the one-dimensional example of Subsection 4.1 three states (in a smeared sense) can be identified: non-fractured, pre-fractured, and fractured. An elastic threshold is indirectly introduced by the truncation in (2.4), so that a non-null activation energy has to be exceeded to pass from the non-fractured to the pre-fractured state. This prevents to have a pre-fracture point with zero jump amplitude which is energetically equivalent to a non-fracture point [25]. In the pre-fractured phase the material exhibits softening, deformation localizes in a narrow region representative of the crack. Then, the fractured state is asymptotically achieved as the damage variable $v \rightarrow 0$.

From the energetic point of view it is observed, in agreement with theory, the transition from a convex to a concave evolution once that the elastic phase is completed. 
Finally, simulations obtained with a decreasing value of the parameter $\delta$ confirm the theoretical $\Gamma$-convergence result of [20].

Analogous results hold for the $C^{1}$ function (2.6), proposed as a regularization of (2.4). We remark that the lack of elastic threshold in this case could be recovered by simply changing the second term of (3.1), according to [40].

The absence of mathematical results in more than one dimension and of a specific formulation does not allow today to investigate with consistency complex problems. However, the first attempts proposed in this paper are very promising. Indeed, the model and the dissipated energy have shown to be mesh independent. Moreover, the $\Gamma$-convergence result demonstrated for scalar displacements seems working even in some special 2D vector-valued cases. We have chosen an example that in a certain sense shares some peculiarities with the $1 \mathrm{D}$ case. The main advantage of the $2 \mathrm{D}$ simulation is the presence of a stress concentrator that deviates the solution from the homogeneous one.

In conclusion we underline that numerical simulations have not only confirmed the analytic results of [20], but have also permitted to study the transition phase of damage evolution and to investigate the peculiarities of the formulation. This puts the basis for further engineering applications.

For what the formulation is concerned, two are the main aspects that must be carefully investigated: unilateral condition and irreversibility. In order to take into account non-interpenetration after fracture closure, a kinematical unilateral constraint should be introduced. As for irreversibility, one aims to analytically identify a memory variable which describes the evolution history in the sharp model, once that damage monotonicity is assumed in the smeared model.

In a forthcoming work the choice of different elastic energy expressions will be investigated in case of looseness and damage of the material micro-structure. The idea follows the lines of [30], where a generic theory of failure is proposed. Simply varying the form of the class for admissible structured deformations, one aims to capture different responses, possibly incorporating the idea of cleavage, deviatoric, combined cleavage-deviatoric, and masonry-like fractures. This latter formulation rigorously avoids material overlapping in the cracked zones and seems to be the best candidate to impose unilateral conditions in a smeared approach.

In the brittle fracture approach the parent parameter $\delta$ assumes a twofold meaning: purely mathematical for $\Gamma$-convergence purpose and physical associated to the intrinsic length scale of the material, thus defining the diffusion zone for damaging process. The first significance is guaranteed by mathematical proofs and numerical validations. The second aspect requires further investigations.

\section{Acknowledgements}

F. Iurlano was supported by the Deutsche Forschungsgemeinschaft through the Sonderforschungsbereich 1060 "The mathematics of emergent effects", project A5. She wishes to acknowledge Sergio Conti for an interesting discussion.

\section{References}

[1] R. Alessi, J.-J. Marigo, and S. Vidoli, Gradient damage models coupled with 
plasticity: Variational formulation and main properties, Mechanics of Materials, 80 (2015), pp. 351-367.

[2] G. Alfano And M. A. Crisfield, Finite element interface models for the delamination analysis of laminated composites: mechanical and computational issues, International Journal for Numerical Methods in Engineering, 50 (2001), pp. 17011736 .

[3] R. Alicandro, A. Braides, and J. Shah, Free-discontinuity problems via functionals involving the $L^{1}$-norm of the gradient and their approximations, Interfaces Free Bound., 1 (1999), pp. 17-37.

[4] M. Ambati, T. Gerasimov, and L. De Lorenzis, Phase-field modeling of ductile fracture, Comput. Mech., 55 (2015), pp. 1017-1040.

[5] — A review on phase-field models of brittle fracture and a new fast hybrid formulation, Computational Mechanics, 55 (2015), pp. 383-405.

[6] L. Ambrosio, N. Fusco, And D. Pallara, Functions of bounded variation and free discontinuity problems, Oxford Mathematical Monographs, The Clarendon Press Oxford University Press, New York, 2000.

[7] L. Ambrosio and V. M. Tortorelli, On the approximation of free discontinuity problems, Boll. Un. Mat. Ital. B (7), 6 (1992), pp. 105-123.

[8] H. Amor, J.-J. Marigo, And C. Maurini, Regularized formulation of the variational brittle fracture with unilateral contact: Numerical experiments, Journal of the Mechanics and Physics of Solids, 57 (2009), pp. 1209 - 1229.

[9] W. Bangerth, R. Hartmann, and G. Kanschat, deal.II - a general purpose object oriented finite element library, ACM Trans. Math. Softw., 33 (2007), pp. 24/1$24 / 27$.

[10] G. I. Barenblatt, The mathematical theory of equilibrium cracks in brittle fracture, in Advances in Applied Mechanics, Vol. 7, Academic Press, New York, 1962, pp. 55-129.

[11] Z. P. Bazant and G. Pijaudier-Cabot, Nonlocal continuum damage, localization instability and convergence., Journal of Applied Mechanics, Transactions ASME, 55 (1988), pp. 287-293.

[12] M. J. Borden, T. J. Hughes, C. M. Landis, and C. V. Verhoosel, A higherorder phase-field model for brittle fracture: Formulation and analysis within the isogeometric analysis framework, Computer Methods in Applied Mechanics and Engineering, 273 (2014), pp. $100-118$.

[13] M. J. Borden, C. V. Verhoosel, M. A. Scott, T. J. Hughes, and C. M. LANDis, A phase-field description of dynamic brittle fracture, Computer Methods in Applied Mechanics and Engineering, 217-220 (2012), pp. 77 - 95.

[14] B. Bourdin, Numerical implementation of the variational formulation for quasistatic brittle fracture, Interfaces Free Bound., 9 (2007), pp. 411-430. 
[15] B. Bourdin, G. Francfort, and J.-J. Marigo, Numerical experiments in revisited brittle fracture, Journal of the Mechanics and Physics of Solids, 48 (2000), pp. $797-826$.

[16] B. Bourdin, G. A. Francfort, and J.-J. Marigo, The variational approach to fracture, J. Elasticity, 91 (2008), pp. 5-148.

[17] B. Bourdin, C. J. Larsen, and C. L. Richardson, A time-discrete model for dynamic fracture based on crack regularization, International Journal of Fracture, 168 (2011), pp. 133-143.

[18] A. Braides, G. Dal Maso, and A. Garroni, Variational formulation of softening phenomena in fracture mechanics: the one-dimensional case, Arch. Ration. Mech. Anal., 146 (1999), pp. 23-58.

[19] M. Cervera, M. Chiumenti, and R. Codina, Mesh objective modeling of cracks using continuous linear strain and displacement interpolations, International Journal for Numerical Methods in Engineering, 87 (2011), pp. 962-987.

[20] S. Conti, M. Focardi, And F. Iurlano, Phase field approximation of cohesive fracture models, Ann. Inst. H. Poincaré Anal. Non Linéaire, (2015).

[21] G. Dal Maso, An introduction to $\Gamma$-convergence, Progress in Nonlinear Differential Equations and their Applications, 8, Birkhäuser Boston Inc., Boston, MA, 1993.

[22] G. Dal Maso and F. Iurlano, Fracture models as $\Gamma$-limits of damage models, Commun. Pure Appl. Anal., 12 (2013), pp. 1657-1686.

[23] G. Dal Maso, G. Orlando, and R. Toader, Fracture models for elasto-plastic materials as limits of gradient damage models coupled with plasticity: the antiplane case, Calc. Var. Partial Differential Equations, 55 (2016), pp. 55-45.

[24] G. Del Piero, One-dimensional ductile-brittle transition, yielding, and structured deformations, in Variations of domain and free-boundary problems in solid mechanics (Paris, 1997), vol. 66 of Solid Mech. Appl., Kluwer Acad. Publ., Dordrecht, 1999, pp. 203-210.

$[25] \_,$A variational approach to fracture and other inelastic phenomena, Journal of Elasticity, 112 (2013), pp. 3-77.

[26] G. Del Piero, G. Lancioni, and R. March, A variational model for fracture mechanics: Numerical experiments, Journal of the Mechanics and Physics of Solids, 55 (2007), pp. 2513-2537.

[27] M. Focardi And F. IURlano, Asymptotic analysis of Ambrosio-Tortorelli energies in linearized elasticity, SIAM J. Math. Anal., 46 (2014), pp. 2936-2955.

[28] G. A. Francfort And J.-J. Marigo, Revisiting brittle fracture as an energy minimization problem, J. Mech. Phys. Solids, 46 (1998), pp. 1319-1342.

[29] F. Freddi And M. Frémond, Damage in domains and interfaces: a coupled predictive theory, J Mech Mater Struct, 1 (2006), pp. 1205-1234. 
[30] F. Freddi and G. Royer-Carfagni, Regularized variational theories of fracture: A unified approach, Journal of the Mechanics and Physics of Solids, 58 (2010), pp. $1154-1174$.

[31] — Variational fracture mechanics to model compressive splitting of masonry-like materials, Annals of Solid and Structural Mechanics, 2 (2011), pp. 57-67.

[32] _ _ Plastic flow as an energy minimization problem. Numerical experiments, Journal of Elasticity, 116 (2014), pp. 53-74.

[33] — Phase-field slip-line theory of plasticity, Journal of the Mechanics and Physics of Solids, 94 (2016), p. 257-272.

[34] A. Giacomini, Ambrosio-Tortorelli approximation of quasi-static evolution of brittle fractures, Calc. Var. Partial Differential Equations, 22 (2005), pp. 129-172.

[35] A. A. Griffith, The phenomena of rupture and flow in solids, Philosophical Transactions of the Royal Society of London, A221 (1921), pp. 163-198.

[36] F. Iurlano, Fracture and plastic models as $\Gamma$-limits of damage models under different regimes, Adv. Calc. Var., 6 (2013), pp. 165-189.

[37] G. Lancioni and G. Royer-CARfagni, The variational approach to fracture mechanics. a practical application to the french panthéon in paris, Journal of Elasticity, 95 (2009), pp. 1-30.

[38] A. Mesgarnejad, B. Bourdin, and M. Khonsari, Validation simulations for the variational approach to fracture, Computer Methods in Applied Mechanics and Engineering, 290 (2015), pp. 420-437.

[39] M. NegRI, Convergence analysis for a smeared crack approach in brittle fracture, Interfaces Free Bound., 9 (2007), pp. 307-330.

[40] K. Pham, H. Amor, J.-J. Marigo, and C. Maurini, Gradient damage models and their use to approximate brittle fracture, International Journal of Damage Mechanics, 20 (2011), pp. 618-652.

[41] B. Schmidt, F. Fraternali, And M. Ortiz, Eigenfracture: an eigendeformation approach to variational fracture, Multiscale Model. Simul., 7 (2009), pp. 1237-1266.

[42] R. Temam, Problèmes mathématiques en plasticité, vol. 12 of Méthodes Mathématiques de l'Informatique [Mathematical Methods of Information Science], Gauthier-Villars, Montrouge, 1983.

[43] D. Wick, T. Wick, R. Hellmig, and H.-J. Christ, Numerical simulations of crack propagation in screws with phase-field modeling, Computational Materials Science, 109 (2015), pp. 367-379. 